\title{
Function of Air-Abrasion Device During Open Flap Surgery in Resective and Regenerative Periodontal Therapy: Case Reports
}

\author{
Orsolya Nemeth*, Fanni Simon, Julia Gango and Marton Kivovics \\ Department of Dental and Oral Surgery, Hungary
}

Received: 制 January 08, 2019; Published: 制 January 17, 2019

*Corresponding author: Németh Orsolya, Dental and Oral Surgery, Educational Institute Address: 1088 Budapest, Szentkirályi utca 40, Hungary

\begin{abstract}
Background: One of the basic pillars of periodontal surgery is open flap surgery, which preserves the regeneration skill of the periodontium. The aim of this case report is to present a novel approach to the effects of airflow device (Varios Combi Pro - NSK) usage during periodontal open flap surgery with a primary focus on periodontal attachment and regeneration. The main goal is to obtain a treated root surface with smooth and hard surface characteristics without endotoxins. Air-abrasive device is an opportunity for root debridement which helps the aim to probing depth reductions and removal of subgingival biofilm.

Description of the Clinical Procedure: The observed group of cases contained of patients with chronic periodontitis, deeper than $4 \mathrm{~mm}$ sockets. Periodontal parameters of each patient had been registered (PPD, GR, CAL, BoP, PI) two days pre-surgery and at the 3-, and 7-months post-surgery appointments. After conservative therapy and motivating our subjects to perform ideal oral hygiene, they were prepared for open flap surgeries. Every case was operated by the same surgical method by contouring flaps, removing the granulation tissues, scaling and root surfaces were conditioned by the air-abrasive device. Besides the same steps, in two cases, Emdogain ${ }^{\circ}$ (Straumann) was applicated into defects.

Description of the Outcomes: After 3 months eventless healing period, the involved teeth showed absence of bleeding on probing. Measurements were always made at six sites per tooth. In two cases the mean pocket depth reduction measured $1.7 \mathrm{~mm}$, in two Emdogain applicated cases reduction were 1.8 and $2.3 \mathrm{~mm}$. The average clinical attachment gain in two cases measured 1.9 and $0.4 \mathrm{~mm}$, while in two cases with Emdogain usage were 4.8 and $3.1 \mathrm{~mm}$. We observed slight $(2 \mathrm{~mm})$ gingival recession. Afterwards 7 months healing period, registered parameters have been improved and we checked a maintained healing procedure. These results indicate that using air-abrasive device provides plaque-free and homogeneous but cemented root surfaces while periodontal ligaments might be properly preserved to give chance for periodontal regeneration.
\end{abstract}

\section{Introduction}

In dentistry, especially in periodontology, wide range of dental materials and equipment's burst upon our view. One of basic pillar of periodontal surgery is open flap surgery, which helps the regeneration of the periodontium. Our working team combined open flap debridement with the usage of air-abrasive treatment for conditioning the root surfaces while we also tried to avoid the total lack of the cementum. The main goal of this procedure was to present the effects of air-polish device (Varios Combi Pro - NSK) usage in surgical treatment to assist periodontal attachment and regeneration

\section{Background}

The major role of cementum by its structural and dynamic qualities is to serve as the site of attachment for principal collagen fibers [1]. Cementoblasts have biological activity and reactivity which perform different functions. They produce two collagencontaining types of cementum with different properties [2]. In periodontal disease, cementum may vary which leads to the loss of connecting tissue attachment to cementum [3]. On periodontally diseased root surfaces bacteria and their endotoxins penetrate into the cementum. It is generally accepted, that removal of plaque and calculus from cementum which infected by pathogenic microorganisms is the main step in periodontal treatment [4]. Then the main goal is to obtain a treated root surface with smooth and hard surface characteristics without endotoxins [5]. In recent studies, air-polishing device has been used as a supplementary treatment besides the open flap surgery procedure. Air-abrasive device is an opportunity for root debridement which helps the aim to probing depth reductions and removal of subgingival biofilm $[6,7]$. 


\section{Description of Clinical Procedure}

Patients with chronic periodontitis, deeper than $4 \mathrm{~mm}$ sockets participated in our 4 cases. Before surgery, all patients received oral hygiene instructions and full mouth supra- and subgingival scaling, in order to reduce the soft tissue inflammation [8]. Following a hygienic phase, 4 female patients aged 40 to 62 years in good general health, non-smokers, are agreed to participate in this case series. After scaling, doing curettages and motivating our group members to perform ideal oral hygiene, we prepared them for open flap surgeries [8]. In order to reduce the mobility when it was needed, the teeth were splinted by steel retainer. Participants have single-rooted front teeth or first molars and have bleeding on probing. Almost every case member has deep intrabony defect with suprabony component. Defects were located in the interproximal area. For the first examination full mouth plaque scores were recorded which revealed the presence of plaque. Each patient periodontal parameters had been registered (PPD, GR, CAL, BoP, PI) 2 days before the surgical procedure. The measurements were implemented on examined teeth, at six locations per tooth using a standardized periodontal probe. Every case was operated with the same surgical method. The patients volunteered for the study after receiving verbal and written information and a signed informed consent approved by Department in Community Dentistry of Semmelweis University, Hungary.

\section{Surgical Procedure}

Patients were asked to rinse with $0.2 \%$ chlorhexidine for 2 minutes before perioral disinfection. Surgeries were performed in local anesthesia. The initial incision was carried out buccal, oral and interproximal intrasulcular incision to the alveolar crest at the experimental site, involving one to two teeth apart. When the wide papilla sizes indicated, we made papilla preservation techniques [9]. A mucoperiosteal flap was elevated to the level of the alveolar crest. After contouring flaps, we had opened view of roots and defects. The granulation tissues were removed, and the defects were fully debrided and root surfaces were scaled by ultrasonic instruments [8]. In the next step, we decontaminated the root surfaces by applying air polish device (Varios Combi Pro perio function) with glycine-based powder (Perio-Mate Powder) $[7,10]$. The flexible nozzle tip can follow tooth contours and it is insertable on root surfaces by reducing the optional periodontal damage. The powder spray pressure is under the supragingival level to provide safe operation during subgingival application. The irrigation of the powder was provided by physiological saline solution [11]. After we had expert view of smooth, homogeneous root surfaces and defects. In two cases when we examined the first molars, we applied enamel matrix derivate (Emdogain $\AA$-Straumann) in three or two walled defects, which predictably regenerates lost periodontal attachment [12].

The product that has been shown to regenerate the cementum, periodontal ligament and alveolar bone in recession defects. Emdogain $₫$ may provide a range of biological effects which are essential in wound healing by the content of peptides with their proteolytic processing [13]. The flaps were united with different types of vertical mattress sutures to obtain positioning of the facial and oral flaps and primary closure of the interdental space over the defects. All patients were post-operatively administered antibiotics for 7 days and painkiller according to individual need. Patients were asked not to brush the surgical area but rinse with $0.2 \%$ chlorhexidine two times daily during the following period of 2 weeks. Sutures were removed at 14 days after surgery. The patients resumed tooth cleaning with the use of a soft brush at following 4 weeks.

\section{Case 1}

A 47-years old woman reported pain and bleeding that occurred while brushing around lower and upper front teeth. During oral examination minimal marginal gingiva hyperplasia was visible. After periodontal status radiograph horizontal bone resorption with vertical components at front and molar area revealed. Patient had narrow and plain papilla's, beside the hyperplastic margin and the patient also had deep suprabony pocket components. Probing pocket depth ranged from 4 to $5 \mathrm{~mm}$. As we sum the treatment up before, non-surgical debridement and oral hygiene reinstruction was provided which leaded to reduction of marginal inflammation. Periodontal parameters registered then the open flap surgery proceeded. In this case we completed the conditioning of root surfaces with air-polishing device as debridement of the cementum.

a) Mean parameters before surgery (Figures 1-6):

b) PPD (mm): 3.1

c) $\quad \mathrm{GR}(\mathrm{mm}): 0$

d) $\quad$ BoP (\%): 65

e) PI (\%): 21

f) CAL: 3.1

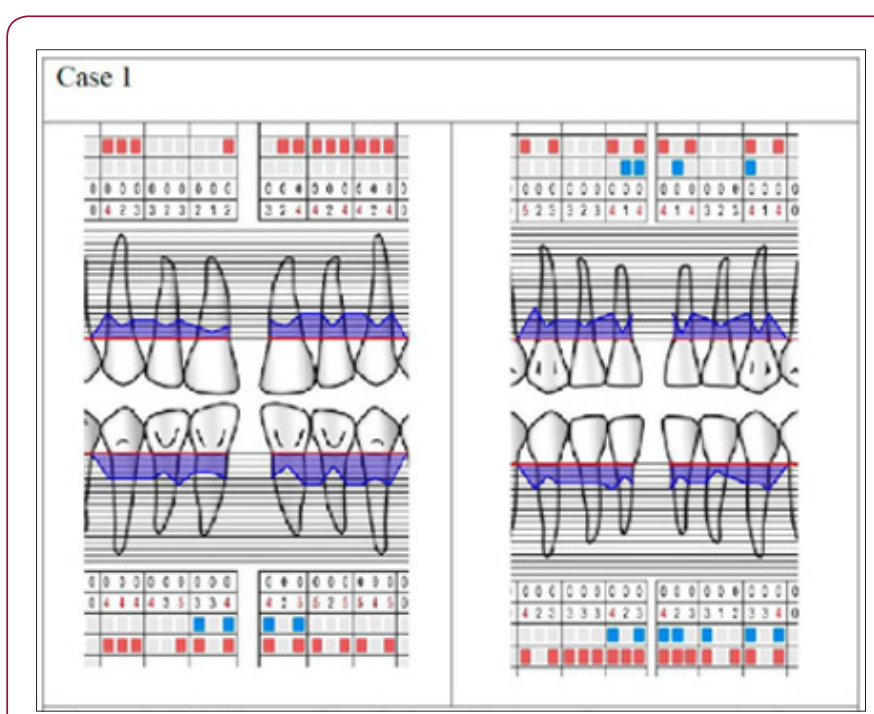

Figure 1: Initial periodontal chart. Registered parameters in the examined areas: lower and upper front teeth. (PPD, GR, BoP, PI). 


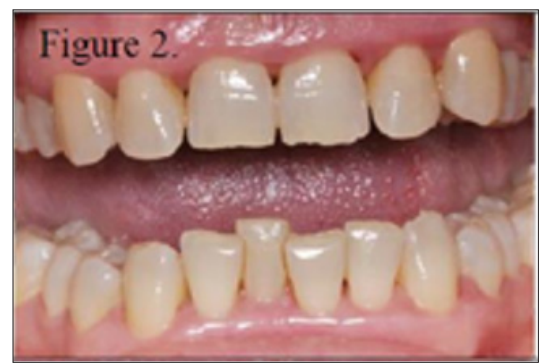

Figure 2: Initial status before surgery.

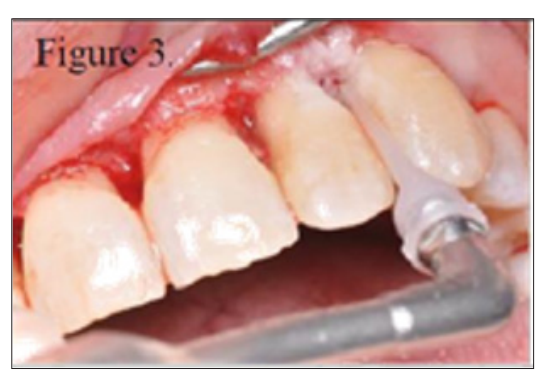

Figure 3: 23 tooth root surface conditioning after debridement.

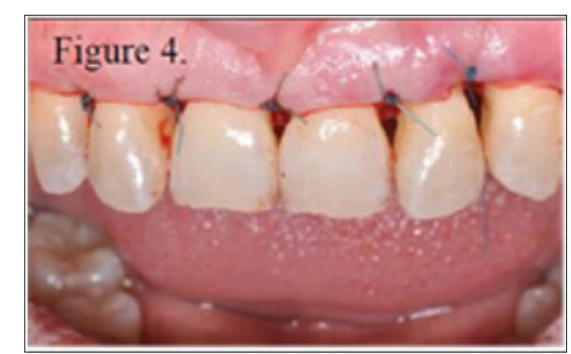

Figure 4: United flaps with modified vertical mattress sutures on upper front region.

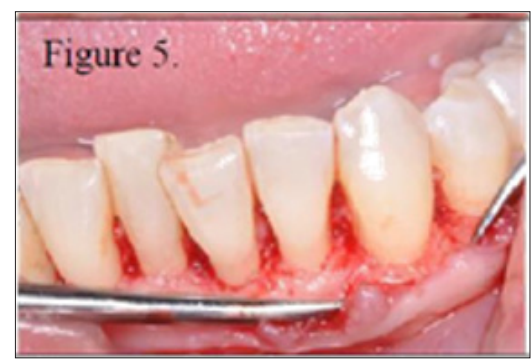

Figure 5: After removing granulation tissue, debrided root conditioning of lower front teeth.

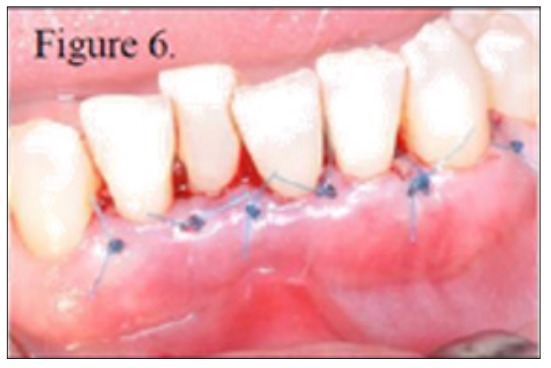

Figure 6: United flaps with vertical mattress sutures on lower front region

\section{Case 2}

A 62-years old woman complained of massive gingiva bleeding on touching entirely of her mouth. The mixture of vertical and horizontal bone loss was detectable on periodontal status radiograph. Because of the bone and the average inflammation, teeth had first class mobility that is why we splinted the upper and lower front teeth before surgery. Besides fixation, we provided supportive non-surgical therapy. During the surgery, we decontaminated the root surfaces with air-abrasion device (Tables $1 \& 2$ ).

Table 1: Results are expressed as the average values of the 4 cases. $\mathrm{PPD}=$ probing pocket depth, $\mathrm{GR}=$ gingival recession, $\mathrm{BoP}=$ bleeding on probing, $\mathrm{PI}=$ plaque index, $\mathrm{CAL}=$ clinical attachment level.

\begin{tabular}{|c|c|c|c|c|c|}
\hline & PPD (mm) & GR (mm) & BoP (\%) & PI (\%) & CAL \\
\hline Case1 & 1.7 & 0.2 & 12 & 0 & 1.9 \\
\hline Case2 & 1.7 & 1.3 & 5 & 8 & 0.4 \\
\hline Case3 & 1.8 & 3 & 0 & 0 & 4.8 \\
\hline Case4 & 2.3 & 0.8 & 0 & 33 & 3.1 \\
\hline
\end{tabular}

Table 2: Results are expressed as the average values of the 4 cases. $\mathrm{PPD}=$ probing pocket depth, GR = gingival recession, $\mathrm{BoP}=$ bleeding on probing, $\mathrm{PI}=$ plaque index, $\mathrm{CAL}=$ clinical attachment level.

\begin{tabular}{|c|c|c|c|c|c|}
\hline $\begin{array}{c}\text { After 7 } \\
\text { months: }\end{array}$ & PPD (mm) & GR (mm) & BoP (\%) & PI (\%) & CAL \\
\hline Case1 & 1.0 & 1.6 & 0 & 0 & 2.6 \\
\hline Case2 & 0.9 & 2.5 & 0 & 0 & 3.4 \\
\hline Case3 & 1.6 & 2 & 0.1 & 0 & 3.6 \\
\hline Case4 & 1.8 & 1.4 & 0 & 0 & 3.2 \\
\hline
\end{tabular}

Average registered parameters (Figures 7-12):

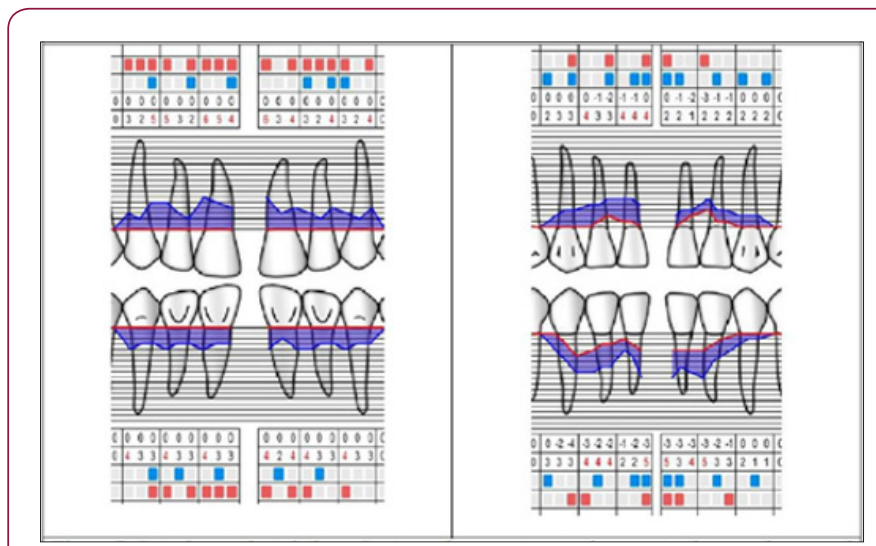

Figure 7: Initial periodontal chart. Registered parameters in the examined areas: lower and upper front teeth (PPD, GR, BoP, PI). 


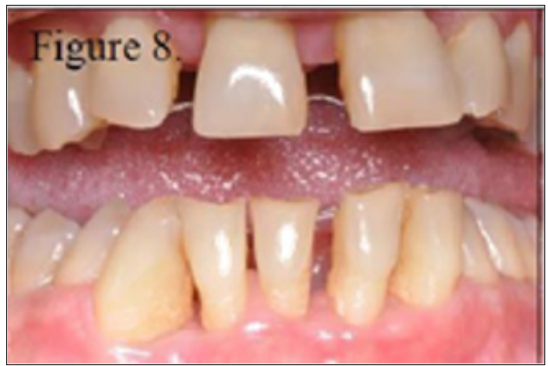

Figure 8: Initial status.

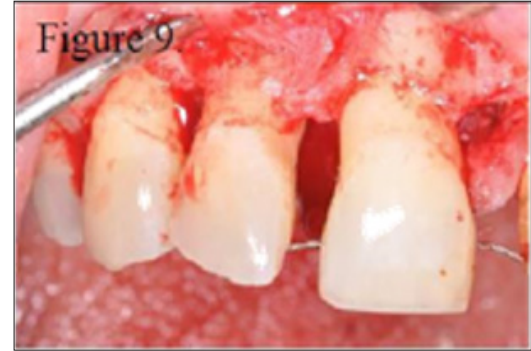

Figure 9: Removal of granulation tissue.

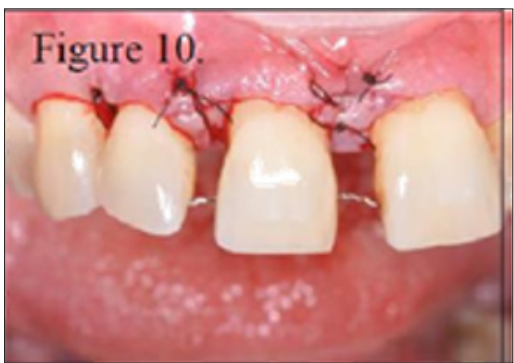

Figure 10: United flaps with modified vertical mattress sutures.

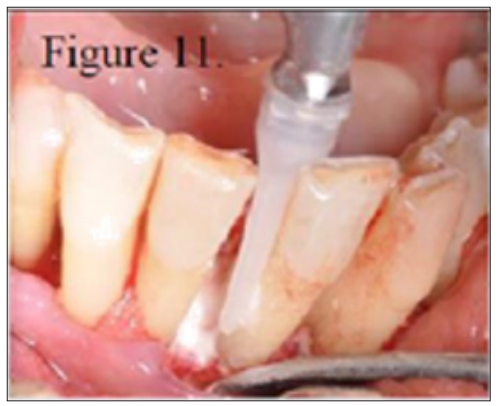

Figure 11: Debrided root conditioning of lower front teeth.

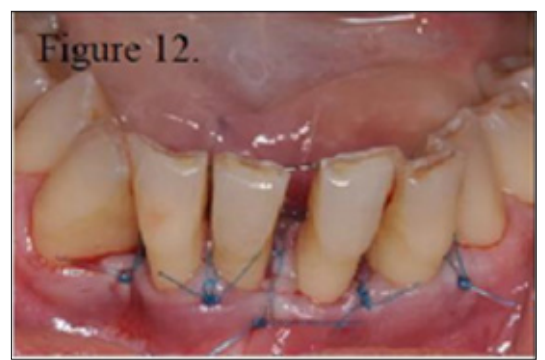

Figure 12: Sutured united flaps.
a) PPD (mm): 3.2
b) GR (mm): 0.5
c) $\quad \mathrm{BoP}(\%): 50$
d) PI (\%): 40
e) CAL: 2.7

\section{Case 3}

A 60-years old woman presents heavy periodontitis, unfortunately we had to extract molar and premolar teeth with third class mobility and suppuration. Thus, the left lower molar quadrant could be prepared for open flap debridement with glycine-powder air-polish supplement. At the mesioapproximal site of the left lower first molar tooth, an average $8 \mathrm{~mm}$ deep 3 walled vertical bone defect was examined preoperatively. After oral hygiene improving, we produced the same open flap operation. We conditioned the root surface in the bone defect with air-polishing device. For the perfect healing and the proper regeneration of the defect we applied enamel matrix protein (Emdogain®-Straumann) [12]. Mean pre-surgery parameters (Figures 13-15):

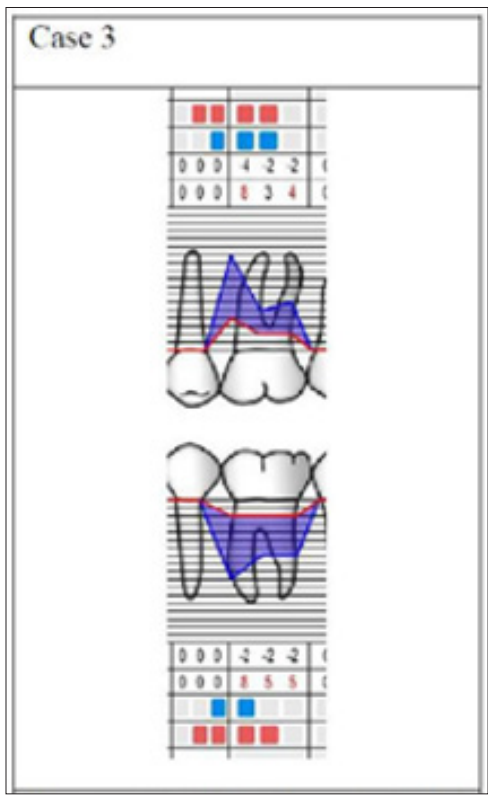

Figure 13: Initial periodontal chart. Registered parameters in the examined areas: lower left first molar (PPD, GR, BoP, PI).

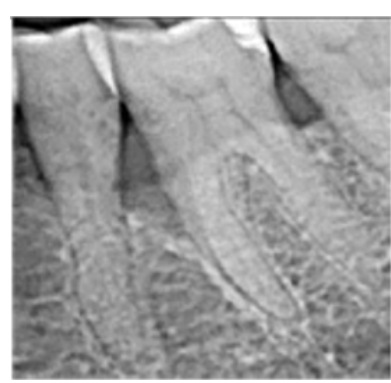

Figure 14: Initial radiograph. 


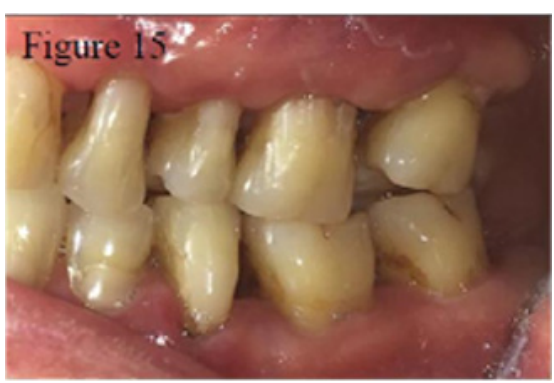

Figure 15: Initial status of lower right first molar.
a) PPD (mm): 5.5
b) GR (mm): 2.3
c) $\mathrm{BoP}(\%): 66$
d) PI (\%): 50
e) CAL: 7.8

\section{Case 4}

A 40-years old woman with moderate periodontitis complained of her right lower first molar because of swelling and bleeding while brushing. During oral examination, a crown was visible on this first molar which had no proper contact with the preparation border of the tooth. At the mesioapproximal site of the first molar, three walled bone defects were diagnosed. Besides this, furcation lesion class-I was detected. For the first step we removed the crown then we accomplished supra- and subgingival scaling and prepared her for the surgery. In this case, we also cleaned the root surface with air-abrasive device then we also applied Emdogain $\AA$ to the defect. During surgery procedure, furcation was degranulated and scaled. Because of the classification of the lesion, we decided to apply Emdogain. The tunneling technique of the furcation lesion was declined, because less than $0.33 \%$ of the furcation was open [12].

Average parameters before surgery (Figures 16-20):

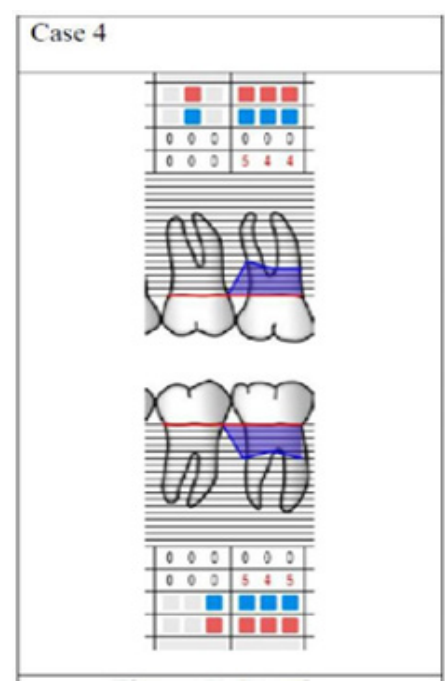

Figure 16: Initial periodontal chart. Registered parameters in the examined areas: lower right first molar (PPD, GR, BoP, PI).

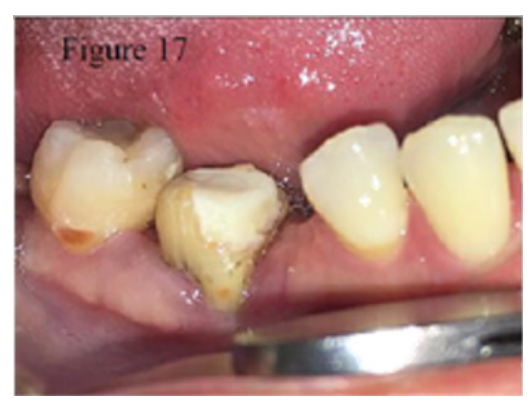

Figure 17: Initial status of lower right first molar.

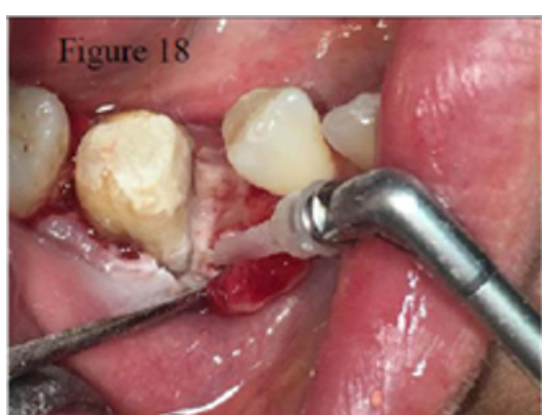

Figure 18: Cleaning the root surfaces with air-abrasive device.

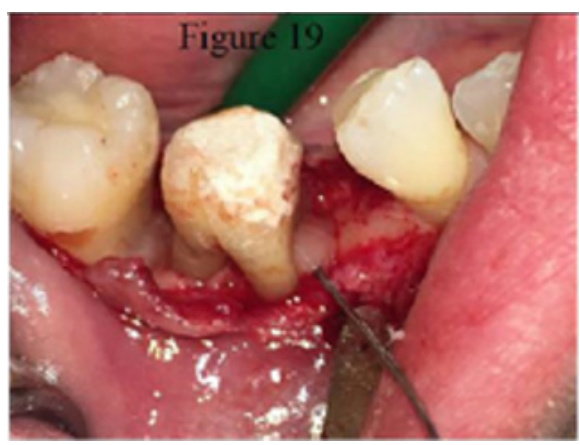

Figure 19: Emdogain application.

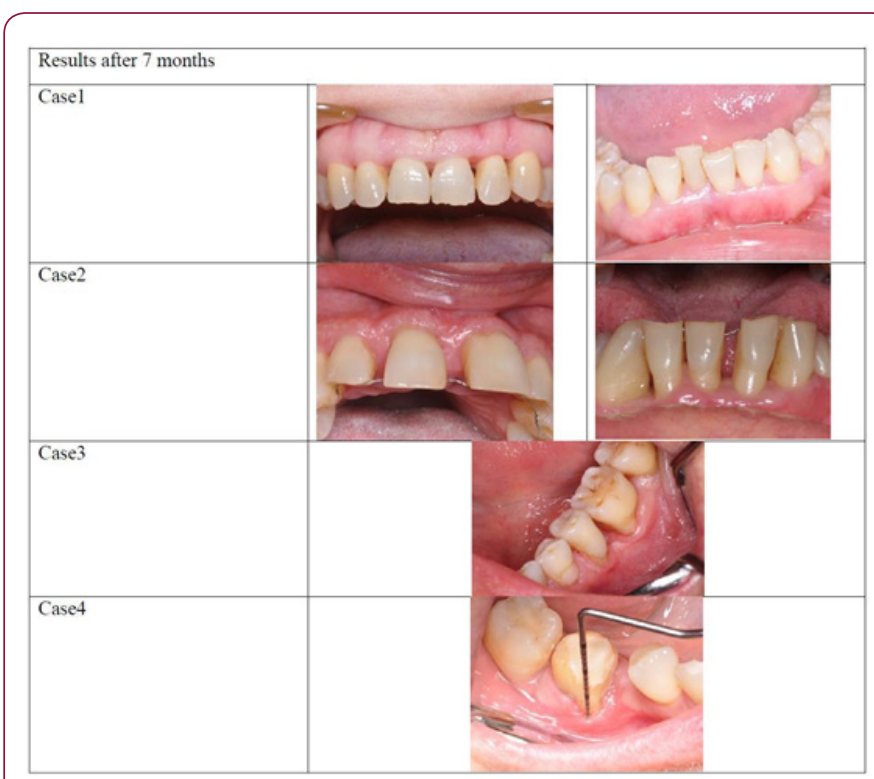

Figure 20: Results of the 4 Cases after 7 months. 

a) PPD (mm): 4.5
b) GR (mm): 0
c) $\operatorname{BoP}(\%): 100$
d) PI (\%): 100
e) CAL: 4.5

\section{Results}

The primary outcome measures for 1 month postoperatively were just visual check-outs to be aware of the proper healing. There was no suppuration or swelling around the operated area, except around Emdogain $®$ applied defects. In these cases, in 1-5 days minimal inflammation were detectable, which had automatically improved. However, as in every case, for Emdogain ${ }^{\circledR}$ supplied operations we prescribed antibiotics post-operative. The following clinical parameters were assessed 3 and 7 months after the surgical procedure using the same type of periodontal probe: probing pocket depth (PPD) gingival recession (GR), bleeding on probing (BoP), plaque index (PI) and clinical attachment level (CAL). Measurements were made at six sites per tooth: mesiobuccal (meb), midbuccal (mb), distobuccal (db), mesiooral (meo), midoral (mo) and distooral (do).

The cemento-enamel junction (CEJ) was used as the reference point. In one case where the CEJ was not visible, a restoration margin was used for these measurements. After 7 months in two cases the mean pocket depth reduction measured 2.1 and $2.3 \mathrm{~mm}$, in two Emdogain applicated cases reduction were 3.9 and $2.7 \mathrm{~mm}$. The average clinical attachment gain in two cases measured 0.5 and $-0.7 \mathrm{~mm}$, while in two cases with Emdogain usage were 4.2 and $1.3 \mathrm{~mm}$. We observed slight $(2 \mathrm{~mm}$ ) gingival recession. The results imply the following in regard to clinical practice: In every cases pocket depth was significantly reduced while gingival recession in merit improved. As a result, clinical attachment level gain was obvious. (Figure 18) Measurements obviously demonstrate that open flap surgery in resective or in regenerative treatment (in our two cases used enamel matrix derivate) with pre-surgical conservative therapy preserve a proper treatment. In these cases, using air-polish device as surface conditioning seemed right supplement without side effects. Figure 18: Results of the 4 Cases after 7 months.

\section{Discussion}

In general, periodontitis has many factors to come into play, so choosing an appropriate treatment can be the utmost concern to every clinician. Patients with general health, progression of the disease and all of the factors should be reflected. In periodontal treatment, non-surgical conservative therapy is the first step or can be a supportive course before surgical phase to achieve posttherapy maintainable periodontal health, so its essential role is obvious.

Surgical side of a treatment is diversified, which means that the basic steps during an open flap surgery can happen by the same methods. Thus, alternative implements are available to complete the different surgical steps, such as surface conditioning, decontaminating, or cleaning. We pursued a different procedure of therapy that utilized air powder abrasion to decontaminate the afflicted site, with occasionally applying regenerative material.

\section{Conclusion}

The result is that, the open flap debridement with air-polish addition revealed in these treatments a well-combined surgical therapy. Although the absence of morbidity and uneventful healing period our cases exhibited suggest that technique described may present a successful process. Based on the results of this report, decontamination in this way can be considered.

\section{Conflict of Interests}

The authors declare that there is no conflict of interests regarding the publication of this paper. The case report was approved by the Regional and Institutional Committee of Science and Research Ethics and the Hungarian Office of Health Authorization and Administrative Procedures and was conducted in accordance with the Declaration of Helsinki.

\section{References}

1. Bosshardt DD, A Sculean (2009) Does periodontal tissue regeneration really work? Periodontology 51(1): 208-219.

2. Nanci A, DD Bosshardt (2006) Structure of periodontal tissues in health and disease. Periodontology 40(1): 11-28.

3. Bosshardt DD, KA Selvig (1997) Dental cementum: the dynamic tissue covering of the root. Periodontology 13(1): 41-75.

4. Polimeni G, AV Xiropaidis, UM Wikesjö (2006) Biology and principles of periodontal wound healing/regeneration. Periodontology 41(1): 30-47.

5. Laleman I, Cortellini S, De Winter S, Rodriguez Herrero E, Dekeyser C, et al. (2017) Subgingival debridement: end point, methods and how often? Periodontology 75(1): 189-204.

6. Mailoa J, Lin GH, Khoshkam V, MacEachern M, Chan HL, et al. (2015) Long-term effect of four surgical periodontal therapies and one nonsurgical therapy: a systematic review and meta-analysis. Journal of periodontology 86(10): 1150-1158.

7. Wennström JL, G Dahlén, P Ramberg (2011) Subgingival debridement of periodontal pockets by air polishing in comparison with ultrasonic instrumentation during maintenance therapy. Journal of clinical periodontology 38(9): 820-827.

8. Horváth A, Stavropoulos A, Windisch P, Lukács L, Gera I, et al. (2013) Histological evaluation of human intrabony periodontal defects treated with an unsintered nanocrystalline hydroxyapatite paste. Clinical oral investigations 17(2): 423-430.

9. Cortellini P, GP Prato, MS Tonetti (1999) The simplified papilla preservation flap. A novel surgical approach for the management of soft tissues in regenerative procedures. International Journal of Periodontics \& Restorative Dentistry 19(6).

10. Caygur A, Albaba MR, Berberoglu A, Yilmaz HG (2017) Efficacy of glycine powder air-polishing combined with scaling and root planing in the treatment of periodontitis and halitosis: A randomised clinical study. Journal of International Medical Research p. 0300060517705540.

11. Flemmig TF, Arushanov D, Daubert D, Rothen M, Mueller G, et al. (2012) Randomized controlled trial assessing efficacy and safety of glycine powder air polishing in moderate-to-deep periodontal pockets. Journal of periodontology 83(4): 444-452. 
12. Sanz M, Jepsen K, Eickholz P, Jepsen S (2015) Clinical concepts for regenerative therapy in furcations. Periodontology 68(1): 308-332.

13. Villa O, Johan C Wohlfahrt, Odd Carsten Koldsland, Steven J Brookes,
Staale P Lyngstadaas, et al. (2016) EMD in periodontal regenerative surgery modulates cytokine profiles: A randomised controlled clinical trial. Scientific reports 6: 23060 .

\section{ISSN: 2574-1241}

DOI: 10.26717/BJSTR.2019.13.002376

Németh Orsolya. Biomed J Sci \& Tech Res

(c) (P) This work is licensed under Creative

Submission Link: https://biomedres.us/submit-manuscript.php

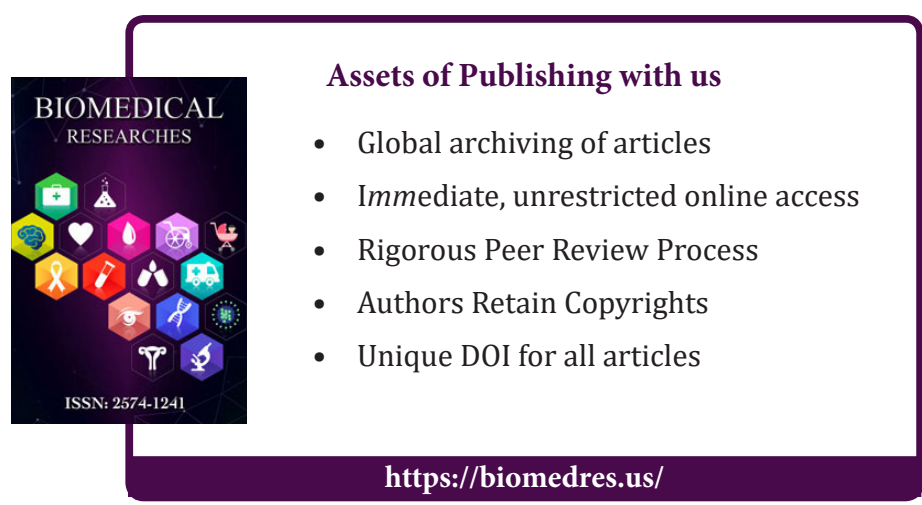

